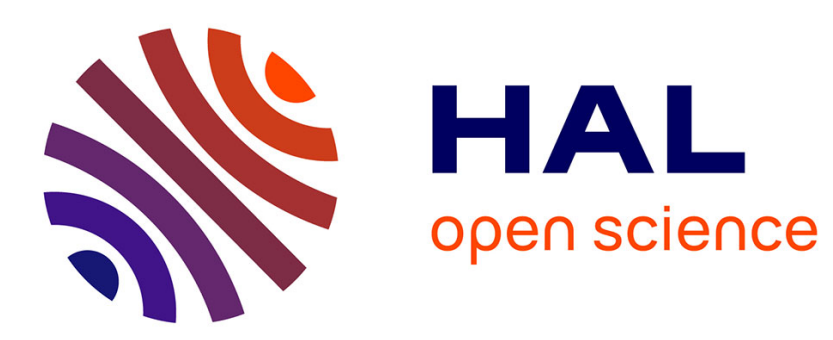

\title{
Network Formation Games for Distributed Uplink Tree Construction in IEEE 802.16j Networks
}

\author{
Walid Saad, Zhu Han, Are Hjorungnes, Merouane Debbah
}

\section{To cite this version:}

Walid Saad, Zhu Han, Are Hjorungnes, Merouane Debbah. Network Formation Games for Distributed Uplink Tree Construction in IEEE 802.16j Networks. IEEE GLOBECOM 2008, Nov 2008, United States. 6 p. hal-00328155

\section{HAL Id: hal-00328155 \\ https://hal-centralesupelec.archives-ouvertes.fr/hal-00328155}

Submitted on 9 Oct 2008

HAL is a multi-disciplinary open access archive for the deposit and dissemination of scientific research documents, whether they are published or not. The documents may come from teaching and research institutions in France or abroad, or from public or private research centers.
L'archive ouverte pluridisciplinaire HAL, est destinée au dépôt et à la diffusion de documents scientifiques de niveau recherche, publiés ou non, émanant des établissements d'enseignement et de recherche français ou étrangers, des laboratoires publics ou privés. 


\title{
Network Formation Games for Distributed Uplink Tree Construction in IEEE 802.16j Networks
}

\author{
Walid Saad ${ }^{1}$, Zhu Han ${ }^{2}$, Are Hjørungnes ${ }^{1}$, and Mérouane Debbah ${ }^{3}$ \\ ${ }^{1}$ UNIK - University Graduate Center, University of Oslo, Oslo, Norway \\ ${ }^{2}$ Electrical and Computer Engineering Department, Boise State University, Boise, Idaho, USA \\ ${ }^{3}$ Alcatel-Lucent Chair in Flexible Radio, SUPÉLEC, Plateau de Moulon, Gif-sur-Yvette, France
}

\begin{abstract}
This paper investigates the problem of the formation of the uplink tree structure among the mobile multi-hop relay (MMR) network's base station (MMR-BS) and its different relay stations (RSs). We model the problem as a network formation game in which the RSs want to form a directed tree graph to improve their utility in terms of the Packet Success Rate (PSR) by using multi-hop cooperative transmission while accounting for a link maintenance cost. In this game, the relay stations engage in bilateral negotiations which result in a contractual agreement to form a directed link between each pair. For network dynamics, we propose a myopic algorithm based on the local best responses of the RSs that converges to a local Nash network. Moreover, the proposed dynamics algorithm allows the RSs to autonomously adapt the network topology to changes in the environment due to mobility or to the presence of heterogeneous traffic. Simulations results show how the RSs can self-organize in a tree structure while improving the network's overall PSR up to $17.5 \%$ and $15.6 \%$ compared, respectively, to the cases where no RSs exist and where the RSs are directly connected to the MMR-BS.
\end{abstract}

\section{INTRODUCTION}

Spatial diversity has proven to be an effective technique for mitigating the fading effects of the wireless channel. Through relaying, mobile nodes cooperate with a given source node in the transmission of its information to a particular destination allowing the source node to benefit from spatial diversity gains. This class of cooperating nodes is referred to as cooperative communications [1] where multiple nodes cooperate to form a virtual multiple antenna array. It has been shown that by using one or multiple relays [1-3] a significant improvement can be witnessed in terms of Bit Error Rate (BER), outage probability and other QoS parameters. For this purpose, the incorporation of cooperative transmission in wireless network standards such as cellular or broadband networks has been under thorough investigation recently. For instance, the IEEE 802.16j Mobile Multi-hop Relay (MMR) task group introduced the concept of multi-hop relaying in the IEEE 802.16 WirelessMAN (WiMAX) family of broadband networks [4]. In 802.16j, a new node, known as the Relay Station (RS) is introduced in the network for the purpose of enhancing capacity and coverage through multi-hop relaying.

The introduction of the RS yields several performance improvements but also faces various challenges. Existing literature tackles several issues related to MMR networks. On one hand, the authors in [5] study the possibility of selecting an optimal position of one RS, which maximizes the total rate, in the presence of one or multiple subscriber stations for the downlink. On the other hand, the work done in [6] investigates the MMR network planning problem with the main aim to find the optimal locations of the MMR-BSs and the RSs for the full deployment of an 802.16j network with minimal monetary costs. The work in [7] provides an algorithm for selecting the optimal downlink path from the MMR-BS to an MS through one or several RS based on a maxi-min throughput criterion. Nevertheless, one promising area in the context of $802.16 \mathrm{j}$ networks, which remains an open problem is the formation of the tree structure connecting the MMR-BS to the RSs in its coverage area [4]. In general, the tree can either be formed at the level of the MMR-BS (centralized approach) or as a result of individual decisions of the RS (distributed approach) [4], [8]. To our knowledge, few existing work has tackled the tree formation problem. The main existing work is done in [8] through a centralized approach that accounts for the link quality between the RSs. Nevertheless, [8] does not account for cooperative transmission on the physical layer. In addition, a centralized approach can yield significant communication overhead, notably in large scale networks with a rapidly changing environment due to RS mobility or incoming heterogeneous traffic.

The main contribution of this paper is to derive a distributed tree formation algorithm for the uplink of an MMR network while accounting for multi-hop cooperative transmission. We model the problem as a network formation game among the RSs. The utility of each RS accounts for the QoS in terms of Packet Success Rate (PSR) as well as the costs for link maintenance. Moreover, we derive a myopic dynamics algorithm for network formation based on local best response strategies of the RSs. In the proposed dynamics, each pair of RSs engage in bilateral negotiations for forming a directed link that can improve the utility of both the initiating and accepting nodes. The dynamics algorithm allows the RSs to self-organize into a local Nash network tree structure rooted at the MMR-BS. Finally, we show how the RSs can autonomously self-organize in order to cope with changes in the environment due to mobility or the presence of heterogeneous data traffic.

The rest of this paper is organized as follows: Section II presents the system model and the game formulation. Section III exposes the properties of the network formation game and presents the proposed dynamics algorithm. Simulation results are presented and analyzed in Section IV. Finally, conclusions are drawn in Section V.

\section{System Model}

\section{A. System Model and Game Formulation}

Consider the uplink of an MMR network with $M$ RSs and one MMR-BS (referred to as BS hereafter). The goal is to propose a distributed algorithm for forming the tree encompassing the BS and all the RSs. Once the uplink tree is formed, the mobile stations (MSs) can connect to the network by selecting a serving RS (or by connecting directly to the BS). We assume that 
each MS deposits its packets at the serving RS through direct transmission. Subsequently, the serving RS acts as a source node and transmits the packets of the MS to the BS through one or multiple hops using cooperative transmission. This assumption on the MSs is considered in order to restrict our attention to the tree formation problem among the RSs which belong to a single WiMAX operator. By doing so, we can propose an algorithm that can be easily integrated within a new or an existing WiMAX network without relying on external entities such as the MSs. For the transmission between each RS and the BS we adopt the decoded relaying multi-hop diversity channel of [3]. In this model, each intermediate node on the path between a transmitting RS and the BS combines, encodes and reencodes the received signal from all preceding terminals before relaying (decode-and-forward). Formally, each $\mathrm{RS} i$ will receive $T_{i}$ packets from all connected MSs, and is required to transmit these packets to the BS through multi-hop relaying along the tree. We also denote by $R_{i}$ the packets received by RS $i$ from connected RSs for relaying purposes. In addition, we assume that all the RSs will continuously transmit one packet, known as a "HELLO" packet in order to maintain their links to the BS active during periods of no activity, i.e., in the absence of MSs. An illustrative example of this system model is shown in Fig. 1.

For modeling the distributed formation of an uplink tree such as in Fig. 1, network formation games provide a suitable framework [9-12]. These games study the interactions among multiple players that want to form a network graph. Each player is considered as a decision maker, and through the various communications among these decision makers a final graph $G$ can form. We model the uplink tree formation problem among the RSs as a network formation game with the RSs being the players. The result of this game is a directed graph $G(V, E)$ formed from $V=\{1, \ldots, M\}$ vertices corresponding to the $M$ RSs and $E$ edges or links. Each link between two RSs $i$ and $j$ denoted $i j \in E$ is a directed connection that goes from RS $i$ to RS $j$ representing the communication flow in the uplink from $i$ to $j$. Each pair of RSs $i$ and $j$ engage in bilateral negotiations, where $\mathrm{RS} i$ suggests to form a link with $j$, and RS $j$ has the opportunity to either accept or reject the offer. If RS $j$ accepts the proposition of $i$, a contract is formed between $i$ and $j$ corresponding to the directed link $i j$. We assume that the BS accepts any connection from any RS, and thus it is considered as an additional fixed vertex in any network graph $G$. This assumption is motivated by the fact that the WiMAX operator deploys the RSs in order to enhance the performance of the BS and thus, the BS should be able to serve all the deployed RSs. Therefore, in addition to the other RSs, a RS can also negotiate a contract with the BS. The strategy space of each $\mathrm{RS}$ is further detailed in Section III. Additionally, we define the notion of path as follows.

Definition 1: A path between two nodes $i$ and $j$ in the graph $G$ is defined as a sequence of nodes $i_{1}, \ldots, i_{K}$ such that $i_{1}=$ $i, i_{K}=j$ and each directed link $i_{k} i_{k+1} \in G$ for each $k \in$ $\{1, \ldots, K-1\}$. We denote the set $Q_{i}$ as the set of all paths from node $i$ to the $\mathrm{BS}$, and thus $\left|Q_{i}\right|$ represents the number of paths from node $i$ to the BS.

Each RS extracts a positive utility from the packets success-

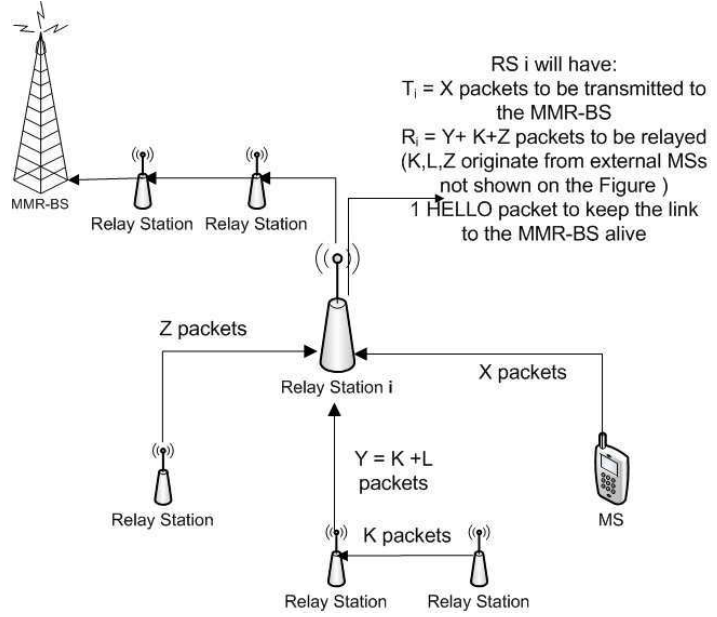

Fig. 1. Example of the uplink tree model.

fully transmitted to the BS out of the $T_{i}$ packets received from external MSs, while accounting for the BER of transmission which constitutes a first cost for transmission. In addition, a RS extracts a positive utility proportional to the number of packets relayed $R_{i}$. The main driver behind this relaying benefit is that the importance of the role of a RS $i$ in the network increases if this RS $i$ serves more RSs and relays more packets, and thus its utility should also increase. Moreover, for each RS $j$, a maintenance cost must be paid for each connection resulting from a link $i j$ directed from RS $i$ to RS $j$. In summary, given a network graph $G$, for each RS $i \in V$ the cost and benefit is captured by the following utility

$$
U_{i}(G)=\alpha_{i} \cdot \frac{\left(T_{i}+1\right)}{\left|Q_{i}\right|} \cdot \sum_{q=1}^{\left|Q_{i}\right|}\left(1-P_{q}^{e}\right)^{K}+\beta_{i} \cdot R_{i}-C\left(L_{i}\right),
$$

where $P_{j}^{e}$ is the BER that RS $i$ achieves while transmitting the $T_{i}$ data packets and 1 HELLO packet to the BS using the decodeand-forward model of [3] encompassing all the RSs connecting $i$ to the BS through the path $q \in Q_{i}$ ( $i$ divides its packets equally among all paths) and $K$ is the size of the packets in bits. Consequently, the term $\left(1-P_{q}^{e}\right)^{K}$ represents the Packet Success Rate (PSR) of the traffic going through each path $q \in Q_{i}$ from $i$ to the BS. $R_{i}$ is the number of packets received by $i$ from other RSs for relaying purposes. $C\left(L_{i}\right)$ is a maintenance cost factor that is an increasing function of the number $L_{i}$ of incident links from other RSs on RS $i . \alpha_{i}$ and $\beta_{i}$ are a price per unit of packets correctly transmitted or relayed; without loss of generality, we assume $\alpha_{i}=\beta_{i}=1$.

For a transmission between a node $V_{1} \in V$ to a destination $V_{n+1}$ (the destination is always the BS, thus $V_{n+1}=B S$ ) going through $n-1$ intermediate relays $\left\{V_{2}, \ldots, V_{n}\right\} \subset V$, denote $N_{r}$ as the set of all receiving terminals, i.e., $N_{r}=\left\{V_{2} \ldots V_{n+1}\right\}$ and $N_{r(i)}$ as the set of terminals that transmit a signal received by terminal $V_{i}$. For a relay $V_{i}$ on the path from the source $V_{1}$ to the destination $V_{n+1}$, we have $V_{r(i)}=\left\{V_{1}, \ldots, V_{i-1}\right\}$. Given this notation, the BER between a source RS $V_{1} \in V$ and the BS destination $V_{n+1}=B S$ will be computed along the path $q=\left\{V_{1}, \ldots, B S\right\} \in Q_{V_{1}}$ using the tight upper bound given in [3] for the decoded relaying multi-hop diversity channel with BPSK modulation as follows 


$$
\begin{aligned}
P_{q}^{e} & \leq \sum_{N_{i} \in N_{r}} \frac{1}{2}\left(\sum _ { N _ { k } \in N _ { r ( i ) } } \left[\prod_{\substack{N_{j} \in N_{r(i)} \\
N_{j} \neq N_{k}}} \frac{\gamma_{k, i}}{\gamma_{k, i}-\gamma_{j, i}}\right.\right. \\
& \left.\left.\times\left(1-\sqrt{\frac{\gamma_{k, i}}{\gamma_{k, i}+2}}\right)\right]\right) .
\end{aligned}
$$

With $\gamma_{i, j}=\frac{P_{i} \cdot h_{i, j}}{\sigma^{2}}$ the average received SNR at node $j$ from node $i$ where $P_{i}$ is the transmit power of node $i, \sigma^{2}$ the noise variance and $h_{i, j}=\frac{1}{d_{i, j}^{\mu}}$ is the path loss with $d_{i, j}$ the distance between $i$ and $j$ and $\mu$ the path loss exponent. Without loss of generality, we assume that all the RSs will transmit with equal power $P_{i}=\tilde{P}, \forall i$. Finally, for a $\operatorname{RS} i$ which is connected to the BS through a direct transmission path $q_{d} \in Q_{i}$ with no intermediate hops, the BER can be given by $P_{q_{d}}^{e}=$ $\frac{1}{2}\left(1-\sqrt{\frac{\gamma_{i, B S}}{2+\gamma_{i, B S}}}\right)$ [2], [3]; with $\gamma_{i, B S}$ is the SNR received at the BS from RS $i$.

\section{B. Cost}

Any well designed maintenance cost function $C\left(L_{i}\right)$ for a $\mathrm{RS} i$ must satisfy several requirements that are needed for adequately modeling the cost for connectivity. For this purpose, we will inspect the part of the utility which shows the rewards and costs on a RS $i$ for accepting a connection from other RSs that is $f\left(L_{i}\right)=R_{i}-C\left(L_{i}\right)$.

First, for any RS $i$, accepting a first link must incur a very low cost $C\left(L_{i}\right)$ and must be feasible for the smallest possible reward in terms of relayed packets $R_{i}$. Thus, we must have $f(1)>0$ for the smallest reward $R_{i}=1$, yielding $C(1)<1$ (obviously $C(0)=0)$, that is a RS $i$ will accept at least one link from other RSs. Second, each RS $i$ can only support a fixed number of connections $\hat{\lambda}_{i}<\lambda_{i}$ from other RSs. The maximum number of connections $\lambda_{i}$ can be configured by the operator in the RSs or it can have a fixed value based on the physical capabilities of the RS. As a result, we must have $f\left(\lambda_{i}\right)=-\infty$ and thus $C\left(\lambda_{i}\right)=$ $+\infty$. The final requirement is that, as the number $L_{i}$ increases, $C\left(L_{i}\right)$ must start increasing slowly and then must reach a steeper increase slope. One function satisfying these requirements is the $\log$ barrier function

$$
C\left(L_{i}\right)=-c_{i} \cdot \log \left(1-\left(\frac{L_{i}}{\lambda_{i}}\right)^{2}\right),
$$

where $\lambda_{i}$ represents the maximum number of connections whereby $C\left(L_{i}\right)=+\infty ; \forall L_{i} \geq \lambda_{i}$ and $c_{i}$ represents a pricing parameter that imposes the minimum relaying benefit required for adding one link (moving from $L_{i}$ to $L_{i}+1$ ). Similarly to $\lambda_{i}$, the pricing parameter $c_{i}$ can be configured in the RSs by the network operator depending on the requirements in terms of minimum packet needs for different $L_{i}$. For a better understanding of the pricing parameter $c_{i}$ consider the rate of increase of $f$ as we move from $L_{i}$ links to $L_{i}+1$ links given by $\Delta f=$ $f\left(L_{i}+1\right)-f\left(L_{i}\right)=\left(R_{i}\left(L_{i}+1\right)-R_{i}\left(L_{i}\right)\right)+\left(C\left(L_{i}\right)-C\left(L_{i}+1\right)\right)$, thus

$$
\Delta f=\Delta R_{i}-c_{i} \cdot \log \left[\frac{\left(1-\left(\frac{L_{i}}{\lambda_{i}}\right)^{2}\right)}{\left(1-\left(\frac{L_{i}+1}{\lambda_{i}}\right)^{2}\right)}\right] .
$$

The minimum number of packets $\Delta R_{i}$ that a $\mathrm{RS} i$ requires in order to accept an incoming connection is given by setting $\Delta f>0$ and thus yielding

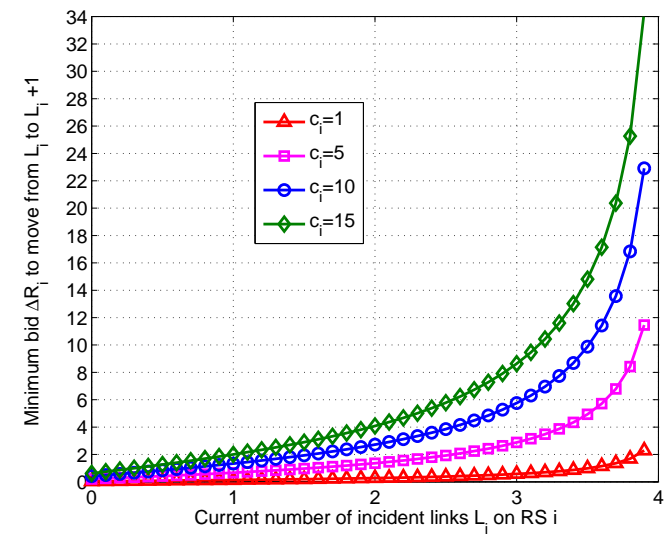

Fig. 2. Minimum required number of packets $\Delta R_{i}$ for RS $i$ in order to accept one additional incoming link (moving from $L_{i}$ to $L_{i}+1$ ) for $\lambda_{i}=5$.

$$
\Delta R_{i}>c_{i} \cdot \log \left[\frac{\left(1-\left(\frac{L_{i}}{\lambda_{i}}\right)^{2}\right)}{\left(1-\left(\frac{L_{i}+1}{\lambda_{i}}\right)^{2}\right)}\right] .
$$

Through (5) we note that, for a fixed maximum number of RS connections $\lambda_{i}$, the pricing parameter $c_{i}$ determines the minimum required number of packets $\Delta R_{i}$ for different $L_{i}$. The relation between $c_{i}$ and $\Delta R_{i}$ is shown in Fig. 2 for $\lambda_{i}=5$. For a given $L_{i}$, as $c_{i}$ increases the minimum $\Delta R_{i}$ required by $\mathrm{RS} i$ increases. For example, for $c_{i}=10 \mathrm{RS} i$ requires 1 packet only to accept a first link $\left(L_{i}=0\right)$ but it requires at least 6 packets to accept a fourth link $\left(L_{i}=3\right)$. Finally, the curve's rate of increase in $L_{i}$ satisfies our requirements and is asymptotic to $L_{i}=4$ since $\hat{\lambda}_{i}<5$ (adding 1 link from $L_{i}=4$ yields an infinite cost).

\section{Network Formation Game: Properties, STRATEGIES AND DYNAMICS}

\section{A. Properties and strategy space of the proposed game}

Several properties of the proposed game can be highlighted. The first property relates to the connectivity of any network graph $G$ formed using our model as follows.

Property 1: Any network graph $G$ resulting from the proposed network formation game will be a connected graph where all the relay stations can communicate through the BS.

Proof: First of all, as mentioned in the previous section, the BS will accept a connection from any RS. Moreover, by inspecting the utility in (1) we notice that if a RS $i$ is not connected to the BS through direct transmission or other multihop paths, the BER will be $P_{q}^{e}=1, \forall q \in Q_{i}$ since $Q_{i}=\emptyset$ (no path from the RS $i$ to the BS) and thus the first term in the utility will be 0 and no RS can improve its utility by disconnecting a link. In other words, a disconnection by any RS will drastically decrease its utility. Therefore, any graph $G$ formed using the proposed game will be a connected graph where all the RSs can communicate through the BS.

Moreover, due to the high disconnection cost, if a RS is unable to find any partner willing to accept forming a link with it, it will connect to the BS through direct transmission. Thus, our network initially starts with all the RSs connected to the BS, before engaging in the network formation game.

As expressed in (1) in the case where a relay is connected to the BS through multiple paths the traffic will be split along these paths. However, after careful inspection of (1), we have the following properties 
Property 2: In the proposed network formation game, each RS $i$ will only have one path $q_{0} \in Q_{i}$ to the BS, thus $\left|Q_{i}\right|=1 \forall i$ and no RS has incentive to form redundant links. As a result, the utility of a RS $i$ reduces to

$$
U_{i}(G)=\left(T_{i}+1\right) \cdot\left(1-P_{q_{0}}^{e}\right)^{L}+R_{i}-C\left(L_{i}\right) .
$$

Proof: By inspecting the first term in the utility of (1), we note that each RS $i$ can improve its utility by directing all of its traffic on the path $q_{0}=\left\{i, i_{2}, \ldots, i_{B S}\right\} \in Q_{i}$ exhibiting the lowest BER. The RS can choose to break all the paths, and keep only $q_{0}$, since relaying more packets will not incur extra costs on the accepting RS $i_{2}$ because $i_{2}$ has already paid the maintenance cost for its connection with $i$ regardless of the traffic that $i$ will deliver (recall that the maintenance cost $C\left(L_{i}\right)$ is a function of the number of RS links and not the amount of traffic). Consequently, RS $i$ benefits by delivering all of its packets to RS $i_{2}$ which provides the smallest BER. Thus, no RS has any incentive to form redundant links and it is easily seen that in this case (1) reduces to (6).

Property 3: Any network graph $G$ resulting from a network dynamics algorithm applied to our network formation game will be a connected tree rooted at the BS.

Proof: This is an immediate consequence of properties 1 and 2. As no RS has the incentive to disconnect from the BS nor to form more than one path to the $\mathrm{BS}$, the final graph resulting from any network dynamics in our game will be a connected tree rooted at the $\mathrm{BS}$.

As a result of these properties, every new link established by a RS $i$ implicitly implies that RS $i$ broke its previous link. Moreover, any network formation dynamics will converge to a tree topology as required by the $802.16 \mathrm{j}$ standard.

Furthermore, we inspect the possible actions or strategies that each RS can take in the proposed game. The strategy space of each RS $i$ consists of the RSs that $i$ wants to connect to (the links that allow $i$ to improve its BER) as well as the links already accepted by $i$ (links directed towards $i$ ). Therefore, the strategy of a RS $i$ is to select the link that it wants to form from the available RSs (or the BS) as well as to select which already accepted links should be maintained. We note that, a RS $i$ cannot connect to a RS $j$ which is already connected to $i$, in the sense that if $j i \in G$, then $i j \notin G$.

Formally, denote $A_{i}=\{j i \mid j \in V-\{i\}\}$ as the set of links that RS $i$ accepted from other RSs and $B_{i}=\{i j \mid j \in$ $\left.(V \cup B S) /\left(\{i\} \cup A_{i}\right)\right\}$ as the set of nodes (RSs or the BS) with whom $i$ wants to connect (note that $i$ cannot connect to RSs that are already connected to it, i.e., RSs in $A_{i}$.). In consequence, each strategy $s_{i}$ of a RS $i$ consists of the pair $s_{i}=\left(a_{i}, b_{i}\right) \in S_{i}=2^{A_{i}} \times B_{i}$, where $2^{A_{i}}$ is the set of all subsets of $A_{i}, a_{i} \in 2^{A_{i}}$ are the accepted links that $i$ wants to maintain, i.e., the links in the set $A_{i} / a_{i}$ will be broken by RS $i$ and $b_{i} \in B_{i}$ is the node with whom $i$ wants to be connected. Note that a RS can only be connected to one other node in our game so selecting to form a link with $b_{i}$ through strategy $s_{i}=\left(a_{i}, b_{i}\right)$ implicitly implies that RS $i$ will replace its previously connected link (if any) with the new link $i b_{i}$. Finally, analogous to the terminology in [12], we refer to all the strategies $s_{i} \in S_{i}$ as local strategies.

Moreover, forming the directed link $i b_{i}$ through a local strategy $s_{i}$ requires the consent of the accepting node $b_{i} \in B_{i}$.
Therefore, RS $i$ does not have the freedom to choose all the possible strategies in the strategy space $S_{i}$ and thus the following definition is required

Definition 2: A local strategy $s_{i}=\left(a_{i}, b_{i}\right) \in S_{i}$ is a feasible local strategy for a RS $i \in V$ if and only if $b_{i}$ is willing to accept a link from RS $i$. Denote $F_{i} \subseteq S_{i}$ as the set of feasible local strategies.

Denote $G_{s_{i}, s_{-i}}$ as the graph $G$ formed when RS $i$ plays a feasible local strategy $s_{i} \in F_{i}$ while all other RSs maintain their vector of strategies $\boldsymbol{s}_{-i}=\left[s_{1}, \ldots, s_{i-1}, s_{i+1}, \ldots, s_{M}\right]$. We define the local best response for a RS as follows [12].

Definition 3: A feasible local strategy $s_{i}^{*} \in F_{i}$ is a local best response for a $\mathrm{RS} i \in V$ if $U_{i}\left(G_{s_{i}^{*}, s_{-i}}\right) \geq U_{i}\left(G_{s_{i}, s_{-i}}\right), \forall s_{i} \in$ $F_{i}$. Thus, the local best response for a $\operatorname{RS} i$ is to make the selection of links that maximizes its utility given that the other RSs maintain their vector of strategies.

\section{B. Dynamics of network formation}

By using the different properties of the RS network formation game, we propose a dynamics algorithm that allows a distributed formation of the network graph. The proposed dynamics assume that each RS is myopic, in the sense that the RSs aim at improving their payoff considering only the current state of the network without taking into account the future evolution of the network. Several models for myopic dynamics have been considered in the network formation literature for directed and undirected graphs [9-12]. In this paper, we propose a myopic dynamics algorithm inspired from [9] and [12]. The proposed algorithm is composed of several rounds where each round mainly consist of two phases: a fair prioritization phase and a dynamics phase. In the fair prioritization phase, we propose a priority function that assigns a priority to each RS. In the dynamics phase, by increasing priority, each RS chooses to play one of its feasible local strategies.

Therefore, each round of the proposed algorithm begins with the fair prioritization phase where each RS is assigned a priority depending on its actual perceived BER: RSs with a higher BER are assigned a higher priority. The motivation behind this procedure is to fairly allow RSs that are perceiving a bad channel to possess an advantage in selecting their partners; for the purpose of improving their BER. Thus, the RSs experiencing a high BER can select their partners out of a larger space of feasible strategies during the dynamics phase. Following prioritization, the RSs start selecting their strategies sequentially. For any strategy $s_{i}=\left(a_{i}, b_{i}\right) \in S_{i}$ that RS $i$ intends to choose, node $b_{i}$ approves to form $i b_{i}$ only if it is able to improve its utility by either adding $i b_{i}$ or replacing one or more of its already accepted links in $A_{b_{i}}$ by $i b_{i}$. Replacing implies that node $b_{i}$ will break one or more of its already accepted links and replace them with $i b_{i}$ if this will improve its utility. Taking these conditions into account, each RS $i$ chooses to play its local best response $s_{i}^{*} \in F_{i}$ (which is a feasible strategy) in order to maximize its utility at each round. In summary, during the dynamics phase, RSs select their local best responses which maximize the utility while taking into account the approval of the accepting nodes in the strategy. Multiple rounds consisting of the above two phases will be run until convergence to the final tree structure $G^{\dagger}$ where 
TABLE I

PROPOSED NETWORK FORMATION DYNAMICS ALGORITHM

Initial State

All the RSs start by directly connecting to the BS.

Two phases in each round of the dynamics

Phase 1 - Fair Prioritization:

Prioritize the RSs from the highest to the lowest current BER.

Phase 2 - Dynamics:

The RSs take action sequentially by priority.

Each RS $i$ plays its local best response $s_{i}^{*}=\left(a_{i}^{*}, b_{i}^{*}\right)$ :

a) RS $i$ selects to maintain the subset $a_{i}^{*} \subseteq A_{i}$ of accepted links, improving its utility by breaking the links in $A_{i} / a_{i}^{*}$.

b) RS $i$ requests to form the link $i b_{i}^{*}$ with node $b_{i}^{*} \in B_{i}$. b.1) If $b_{i}^{*}$ is a RS, it accepts to form $i b_{i}^{*}$ through add or replace ( $s_{i}^{*}$ is a feasible strategy, see definitions 2 and 3$)$.

i- $b_{i}^{*}$ adds $i b_{i}^{*}$ if doing so improves it utility or

ii- $b_{i}^{*}$ replaces one ore more of its current links with $i b_{i}^{*}$ if doing so improves it utility.

b.2) If $b_{i}^{*}$ is the BS, it accepts to form $i b_{i}^{*}$ through add.

Multiple rounds are run until convergence to the final local Nash tree $G^{\dagger}$ where no RS can improve its utility by a unilateral change of strategy.

the RSs can no longer improve their utility through local best responses. The proposed algorithm is summarized in Table I. The stability of the final graph $G^{\dagger}$ is given using the following concept [12]

Definition 4: A network graph $G$ in which no node $i$ can improve its utility by a unilateral change in its local feasible strategy $s_{i} \in F_{i}$ is a local Nash network.

Alternatively to the above definition, a local Nash network is a network where the links chosen by each node are the local best responses. The local Nash network is thus a network where the nodes are in a Nash equilibrium with no node able to improve its utility by unilaterally changing its current local strategy. When our dynamics converge, we have

Lemma 1: The final tree structure $G^{\dagger}$ resulting from the proposed dynamics is a local Nash network.

Proof: In each round of the proposed algorithm, each RS selects the local best response that maximizes its utility in this round. The proposed algorithm converges when no individual RS $i$ can improve its utility by unilaterally changing its strategy $s_{i}$. Thus, at the convergence, the RSs are in a Nash equilibrium resulting from playing the local best responses and the final tree structure $G^{\dagger}$ resulting from the proposed dynamics is a local Nash network.

The proposed algorithm can be implemented in a distributed manner. For choosing the local best response $s_{i}^{*}=\left(a_{i}^{*}, b_{i}^{*}\right)$, each RS can locally assess its current contracts and choose the subset $a_{i}$ that it wants to maintain while breaking the rest. In addition, each RS can easily survey neighboring RSs and acquire the BER that each neighbor can provide. As a result, each RS can take an individual decision to select the node $b_{i}^{*}$ that can maximize its utility; without relying on any centralized entity. The signaling required for gathering this information can be minimal as each RS can easily measure its current channel towards the BS and feed it back to the neighboring RSs. Finally, in order to allow the RSs to adapt to mobility as well as to the presence of MSs; the proposed algorithm will periodically run, allowing the RSs to self-organize and cope with these environmental changes.

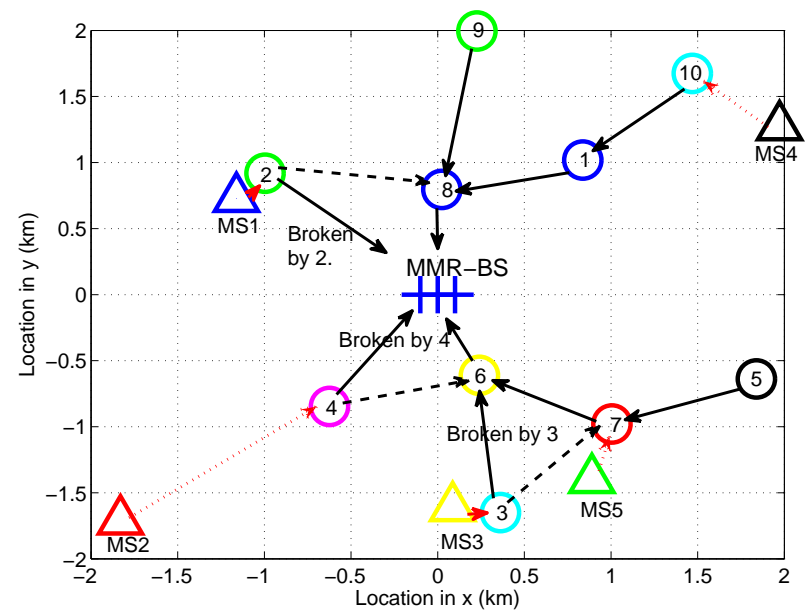

Fig. 3. Snapshot of a tree topology formed using the proposed algorithm with 10 RSs before and after the deployment of 5 MSs.

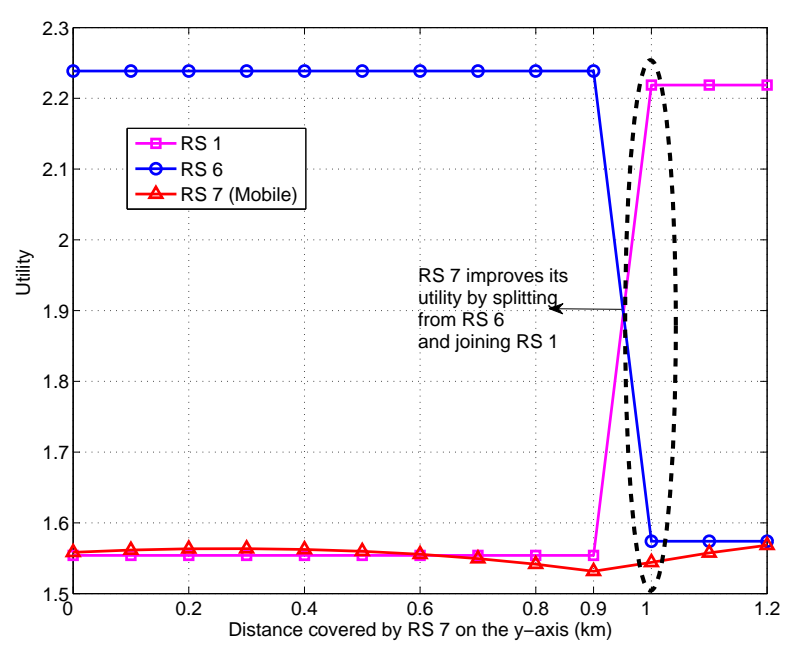

Fig. 4. Self-adaptation of the network's tree topology to mobility shown through the variation of the utility of RS 7 as it moves upwards on the y-axis.

\section{Simulation Results And Analysis}

For simulations, we consider a square area of $2 \mathrm{~km} \times 2 \mathrm{~km}$ with the BS at the center. We deploy the RSs and the MSs within this area. The transmit power is $\tilde{P}=0.01 \mathrm{~W}$, and the noise level is $-110 \mathrm{dBm}$. The propagation loss is $\alpha=3$ and the packet length is $K=128$ bits. The maximum number of RS connections per RS $i$ is $\lambda_{i}=5, \forall i \in V$ and the pricing parameter $c_{i}=10, \forall i \in V$.

In Fig. 3 we randomly deploy $M=10$ RSs within the BS area and all these RSs start by connecting directly to the BS. As a first step, we run the proposed algorithm prior to the presence of any MS in the network (the case where each RS has only 1 HELLO packet and $T_{i}=0 \forall i$ ) and the final local Nash structure that forms in this step is shown in the figure by the solid arrows between the RSs. Moreover, through this figure we can clearly see how the proposed algorithm proceeds before any MS enters the network. For example, RS 4 connects itself directly to the BS although it is closer to RS 6. This is due to the fact that the priority of RS 4 is lower than that of RSs 3 and 7, thus the bidding turn of RS 4 on the resources of RS 6 follows that 
of 3 and 7. As a result, when RS 4 bids to connect to RS 6; the latter had already accepted two links from RSs 3 and 7 and will not accept a third unless this third can satisfy the minimum requirement of 3 packets (Fig. 2). However, RS 4 cannot satisfy this bid and thus, it remains connected to the BS since it is unable to find any other alternative to improve its utility. This example shows how the initial tree forms through our dynamics.

After the initial tree structure is formed, 5 MSs having 5 packets each are randomly deployed as shown in Fig. 3 (each MS connects to the closest RS). Once this additional traffic enters the network, the RSs can self-organize and modify the topology in order to efficiently cope with the incoming traffic. The changes in the topology are shown with labels (broken links) and dashed lines (newly formed links) in Fig. 3. For instance, RSs 2 and 4 are able to break their existing connection with the BS and improve their QoS by connecting to RSs 8 and 6 respectively. Prior to the presence of the MS traffic on RSs 2 and 4, they could not satisfy the minimum required packets for connecting to RSs 8 and 6 respectively. Moreover, we note that RS 3 is also able to improve its utility by breaking its contract with 6 and connecting to RS 7. In a nutshell, Fig. 3 summarizes the operation of the proposed dynamics algorithm with and without the presence of external MS data traffic.

Furthermore, we assess the effect of mobility on the network structure. We consider the network of Fig. 3 prior to the deployment of the MSs and we assume that RS 7 is moving upwards on the positive $y$-axis while the other RSs remain fixed. The changes in utility of the concerned RSs during the movement of RS 7 are shown in Fig. 4. As RS 7 moves upwards; it gets closer to the BS and starts by improving its utility. As it moves around $0.3 \mathrm{~km}$; RS 7 distances itself from RS 6 and thus its utility starts to drop. As it moves around $1 \mathrm{~km}, \mathrm{RS} 7$ is able to improve its utility by disconnecting the link with RS 6 and connecting to RS 7. We note that when RS 7 splits from 6; the utility of RS 6 drops while that of RS 1 improves due to the additional packets for relaying provided by RS 7 . Through these results, the operation of the algorithm in the presence of mobile RSs is illustrated.

Finally, we inspect the average overall network's PSR achieved at the BS by all the MSs. We compare the performance with the case with no RSs and the case where the RSs are directly connected to the BS (no tree). We randomly deploy two classes of MSs with equal probability, a first class representing medium traffic with 5 packets per MS and a second class representing heavy traffic with 10 packets per MS. The results are averaged over random positions of the MSs and the RSs and are shown in Fig. 5 for different numbers of MSs and RSs. In this figure, as the number of MSs increases, for all the schemes, the PSR increases and then stabilizes to a constant maximum. Furthermore, as the number of RSs increases, the performance advantage of our algorithm, in terms of PSR, increases significantly at all network loads. The proposed algorithm presents, respectively, an overall PSR improvement up to $17.5 \%$ and $15.6 \%$ over the schemes without RSs and with direct RS-BS connection.

\section{CONCLUSIONS}

This paper proposed a novel distributed approach to the uplink tree formation problem in $802.16 \mathrm{j}$ networks through a

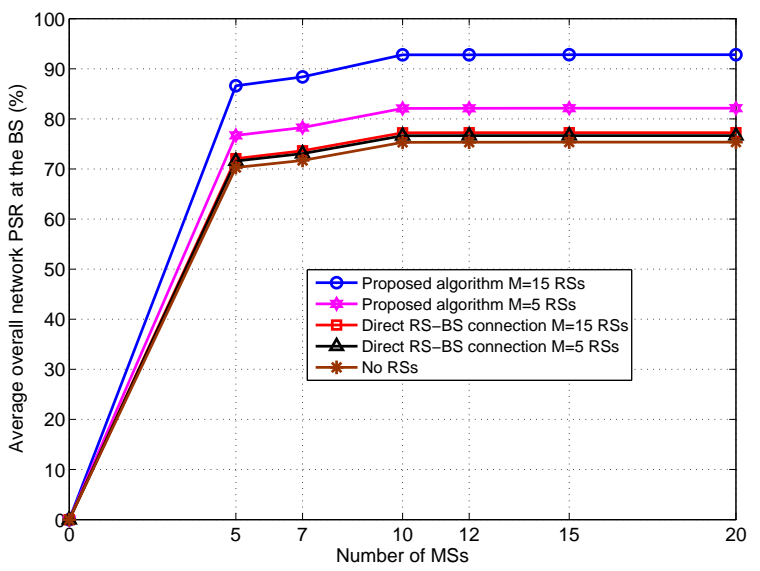

Fig. 5. Total PSR at the BS for different number of RSs and MSs.

network formation game model. In the proposed game, the RSs engage in pairwise negotiations for forming directed links that result in a final tree structure rooted at the BS. The proposed model accounts for the PSR gains from cooperative decode-andforward transmission as well as the costs for link maintenance. For modeling the interactions between the RSs, a distributed myopic dynamics algorithm is derived. The algorithm enables the RSs to maximize their utility while forming a local Nash network. Through simulations, we showed how the RSs are able to autonomously self-organize, adapting the topology to environment changes due to mobility and heterogeneous traffic while improving the overall network's PSR.

\section{REFERENCES}

[1] J. Laneman, D. Tse, and G. Wornell, "Cooperative diversity in wireless networks: efficient protocols and outage behavior," IEEE Trans. Inform. Theory, vol. 50, pp. 3062-3080, Dec. 2004.

[2] A. Sadek, W. Su, and K. R. Liu, "Multinode cooperative communications in wireless networks," IEEE Trans. Signal Processing, vol. 55, pp. 341-355, Jan. 2007.

[3] J. Boyer, D. Falconer, and H. Yanikomeroglu, "Multihop diversity in wireless relaying channels," IEEE Trans. Commun., vol. 52, pp. 18201830, Oct. 2004.

[4] The Relay Task Group of IEEE 802.16, “The p802.16j baseline document for draft standard for local and metropolitan area networks," $802.16 \mathrm{j}$ 06/026r4, Tech. Rep., Jun. 2007.

[5] B. Lin, P. Ho, L. Xie, and X. Shen, "Optimal relay station placement in IEEE 802.16j networks," in Proc. International Conference on Communications and Mobile Computing, Hawaii, USA, Aug. 2007.

[6] Y. Yu, S. Murphy, and L. Murphy, "Planning base station and relay station locations in IEEE 802.16j multi-hop relay networks," in Proc. IEEE Consumer Communications and Networking Conference, Las Vegas, USA, Jan. 2008.

[7] D. M. Shrestha, S. Lee, S. Kim, and Y. Ko, "New approaches for relay selection in IEEE 802.16 mobile multi-hop relay networks," in Proc. EuroPar conference, Rennes, France, Aug. 2007.

[8] H. Lee, H. Park, Y. Choi, Y. Chung, and S. Rhee, "Link adaptive multihop path management for IEEE 802.16j," in IEEE C802/16j-07/1053, Jan. 2007.

[9] M. O. Jackson and A. Watts, "The evolution of social and economic networks," Journal of Economic Theory, vol. 106, pp. 265-295, Nov. 2002.

[10] G. Demange and M. Wooders, Group formation in economics: networks, clubs and coalitions. Cambrdige, UK: Cambrdige University Press, Mar. 2005.

[11] R. Johari, S. Mannor, and J. Tsitsiklis, "A contract based model for directed network formation," Games and Economic Behavior, vol. 56, pp. 201-224, Jul. 2006.

[12] J. Derks, J. Kuipers, M. Tennekes, and F. Thuijsman, "Local dynamics in network formation," http://www.math.unimaas.nl/PERSONAL/jeand/downlds/derdyn.pdf, Maastricht University, Department of Mathematics, Dec. 2007. 